\title{
Presión intraperitoneal y ultrafiltración conseguida con diferentes volúmenes intraperitoneales
}

\author{
Lucila Fernández Arroyo르, Ana Dorado García², Berta María Martín Alcón ${ }^{3}$, Isabel Casanova Mateo², Alicia \\ Quintano Pintado², María Jesús Rollán de la Sota ${ }^{1}$, Mercedes Domínguez Gómez³. \\ ${ }^{1}$ Hospital Clínico Universitario de Valladolid. ${ }^{2}$ Hospital Universitario Río Hortega. Valladolid. ${ }^{3}$ Complejo Asistencial \\ de Palencia. España
}

\section{Resumen}

En condiciones fisiológicas el abdomen actúa como una cavidad cerrada cuya presión aumenta de forma proporcional al volumen que contiene.

El valor normal de la presión hidrostática intraperitoneal está alrededor de $0 \mathrm{y}$ en pacientes en diálisis peritoneal con volúmenes intraperitoneales de 2 litros puede estar en torno a $12 \pm 2 \mathrm{cmH}_{2} \mathrm{O}$.

Realizamos un estudio multicéntrico cuantitativo, descriptivo, longitudinal y prospectivo, que incluía a 42 pacientes en programa de diálisis peritoneal con el fin de examinar la presión intraperitoneal y la ultrafiltración conseguida al infundir diferentes volúmenes de líquido de diálisis. Se hicieron dos intercambios consecutivos el primero con $2500 \mathrm{ml}$ y el segundo con $1500 \mathrm{ml}$ con líquido de diálisis con glucosa $2,3 \%$ y permanencia de 120 minutos en cada intercambio. De los 42 pacientes el $71,5 \%$ eran hombres, con una edad de $59.31 \pm 12.23$ años y con un índice de masa corporal de $27.01 \pm 4.46$. La presión intraperitoneal con volumen intraperitoneal 0 fue de $8.2 \pm 4.1$; con volumen intraperitoneal $2500 \mathrm{ml}$ la presión fue de $13.8 \pm 4.4$ y la ultrafiltración de $131 \pm 206$; con volumen intraperitoneal de $1500 \mathrm{ml}$ la presión fue

Correspondencia:

Lucila Fernández Arroyo

Servicio Nefrología. Diálisis Peritoneal

Hospital Clínico Universitario Valladolid

Avda. Ramón y Cajal, 3.47003 Valladolid. España

E-mail: luci.f.a@hotmail.com de $11.2 \pm 4.2$ y la ultrafiltración de $192 \pm 145$. La ultrafiltración con respecto a la infusión fue del $5,2 \% \pm 8,2 \%$ con volumen de $2500 \mathrm{ml}$ y del $12,8 \% \pm 9,6 \%$ con volumen de $1500 \mathrm{ml}$. Podemos describir un aumento de la presión intraperitoneal al aumentar el volumen intraperitoneal, al mismo tiempo hemos observado una mayor ultrafiltración con volúmenes más bajos que implicaban a su vez cifras menores de presión.

PALABRAS CLAVE:

- PRESIÓN INTRAPERITONEAL

- ULTRAFILTRACIÓN

- VOLUMEN INTRAPERITONEAL

Intraperitoneal pressure and ultrafiltration achieved with different intraperitoneal volumes

\section{Abstract}

Under physiological conditions the abdomen acts as a closed cavity whose pressure increases proportionally to the volume contained.

The normal value of intraperitoneal hydrostatic pressure is around 0 and in peritoneal dialysis patients with intraperitoneal volumes of 2 liters can be around $12 \pm 2 \mathrm{cmH}_{2} \mathrm{O}$.

A quantitative, descriptive, longitudinal, and prospective multicentric study was conducted with 42 patients on a peritoneal dialysis program to examine intraperitoneal pressure and ultrafiltration achieved 
by infusing different volumes of dialysis fluid. Two consecutive exchanges were made: the first with 2500 $\mathrm{ml}$ and the second with $1500 \mathrm{ml}$; both with dialysis fluid with glucose $2.3 \%$ and permanence of 120 minutes in each exchange.

Of the 42 patients, $71.5 \%$ were men, with a mean age of $59.31 \pm 12.23$ years and a body mass index of $27.01 \pm$ 4.46. The intraperitoneal pressure with intraperitoneal volume 0 was $8.2 \pm 4.1$; with the intraperitoneal volume of $2500 \mathrm{ml}$ the pressure was $13.8 \pm 4.4$ and the ultrafiltration of $131 \pm 206$; with the intraperitoneal volume of $1500 \mathrm{ml}$ the pressure was $11.2 \pm 4.2$ and the ultrafiltration of $192 \pm 145$.

Ultrafiltration with respect to infusion was $5.2 \%$ $\pm 8.2 \%$ with a volume of $2500 \mathrm{ml}$ and of $12.8 \%$ $\pm 9.6 \%$ with a volume of $1500 \mathrm{ml}$. We can describe an increase in intraperitoneal pressure by increasing the intraperitoneal volume; at the same time we have observed a higher ultrafiltration with lower volumes which, in turn, implied lower values of pressure.

\section{KEYWORDS}

- INTRAPERITONEAL PRESSURE

- ULTRAFILTRATION

- INTRAPERITONEAL VOLUME

\section{Introducción}

La cavidad peritoneal es una cavidad virtual cerrada cuya presión aumenta de forma proporcional al volumen que contiene. En condiciones normales este volumen está en torno a $50 \mathrm{ml}$ con una tasa de recambio de 1 litro al día ${ }^{1}$.

La presión hidrostática intraperitoneal (PIP) no tiene una diferencia estadísticamente significativa con la presión abdominal ${ }^{2}$ y su valor normal es equivalente o ligeramente inferior a la atmosférica, considerando rangos normales entre 0 y $7 \mathrm{cmH}_{2} \mathrm{O}$, esta presión puede variar con la tos, el estornudo, la respiración, las maniobras de Valsalva, el embarazo, la ascitis y la diálisis peritoneal ${ }^{3,4,5}$.

Varios trabajos confirman que el tamaño del paciente, el tono muscular, su posición o el volumen de la solución de diálisis también modificarán de forma significativa la presión intraabdominal6, ${ }^{6,7}$.
En pacientes en diálisis peritoneal (DP) con volúmenes intraperitoneales de 2 litros la PIP puede estar en torno a $12 \pm 2 \mathrm{cmH}_{2} \mathrm{O}$. El aumento de la presión es lineal con respecto al volumen intraperitoneal siendo significativo cada $500 \mathrm{ml}$ de líquido de diálisis ${ }^{1,8}$.

Por consenso se define la hipertensión intraabdominal como el aumento patológico, persistente o repetido de la $\mathrm{PIP} \geq 12 \mathrm{cmH}_{2} \mathrm{O}$ y se considera que no se debería sobrepasar los $20 \mathrm{cmH}_{2} 0^{9}$, este aumento de la PIP tiene efectos sobre la función cardiovascular y pulmonar, a nivel hormonal y puede ser determinante en problemas de la pared abdominal como hernias y fugas $^{1,7,10,11,12}$.

La PIP también influye en la ultrafiltración (UF) aumentando la reabsorción linfática y la absorción de líquidos a los tejidos adyacentes. Con PIP demasiado elevadas se ha descrito una disminución del volumen ultrafiltrado que se resuelve disminuyendo el volumen de líquido de diálisis (LD) reduciendo de esta forma la presión ${ }^{13,14,15,16}$.

La medida de la PIP de forma sistemática no está recomendada en las guías salvo en pacientes pediátricos pero la información que nos proporciona hace que sea una práctica útil para individualizar el tratamiento de nuestros pacientes y detectar posibles complicaciones de forma precoz $z^{7,17,18}$.

El objetivo del presente estudio es examinar la PIP y la UF conseguida al infundir diferentes volúmenes de LD en pacientes en diálisis peritoneal.

\section{Material y método}

Estudio multicéntrico cuantitativo, descriptivo, longitudinal y prospectivo.

Se incluyeron a 42 pacientes en programa de diálisis peritoneal de las dos unidades que existen en Valladolid y en la unidad de DP de Palencia. Incluimos a todos los pacientes en programa durante el segundo semestre del 2015 y el primer trimestre del 2016, todos los pacientes llevaban al menos mes y medio en programa y no habían padecido intervenciones quirúrgicas abdominales en los 3 meses anteriores. Las causas de exclusión fueron la negación a participar en el estudio y la no tolerancia al volumen máximo infundido.

Los pacientes permanecieron en régimen de estancia en hospital de día durante todo el procedimiento. 


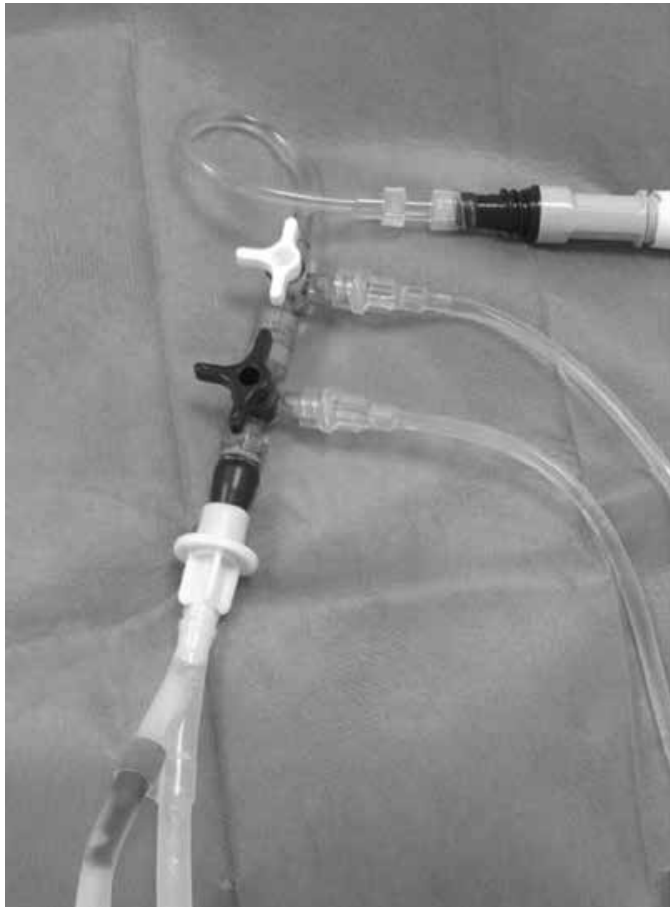

Fig. 1. Conexión utilizada para medición.

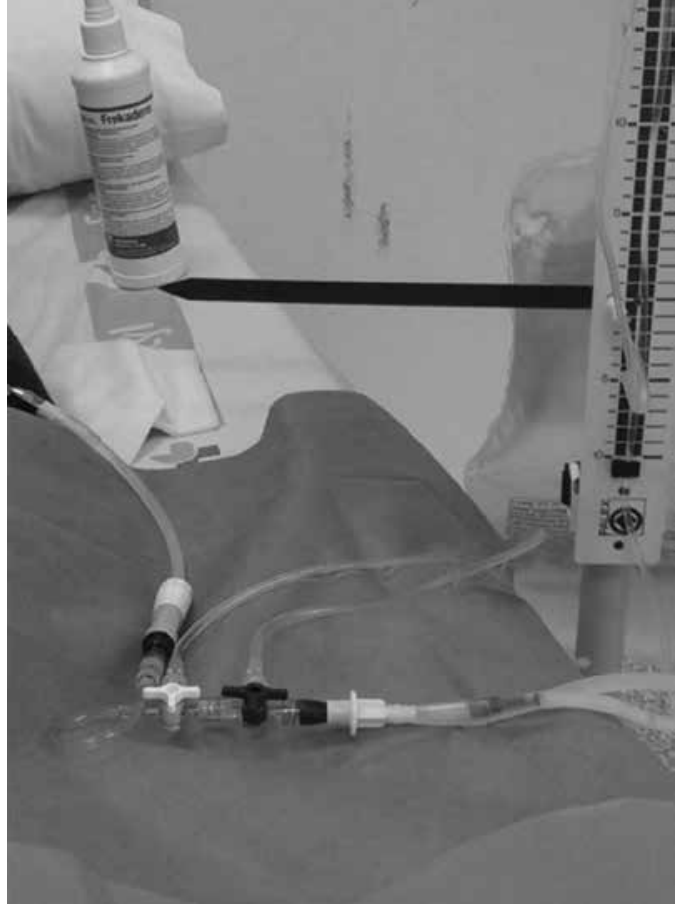

Fig. 2. Conexión y regleta de medida.
Se obtuvo la aprobación de los comités de ética y de investigación, así como el consentimiento informado de cada paciente.

Para realizar el estudio se diseñó un protocolo de actuación que describía la técnica y una gráfica para recoger todas las variables incluidas: edad, sexo, peso, talla, índice de masa corporal (IMC), superficie corporal (SC), PIP, volumen de líquido infundido, volumen de líquido drenado, tiempo de permanencia y ultrafiltración.

Durante todo el procedimiento se extremaron las medidas de asepsia al realizar las conexiones. En ocasiones fue necesario el recambio del equipo prolongador del catéter. Se emplearon adaptadores con conexión luer lock, para unir al catéter el equipo utilizado para medir la presión intraperitoneal y la línea del sistema de diálisis. (Fig. 1 y 2).

Para medir la PIP se usó un sistema con regla métrica y columna de líquido. Antes de realizar cada medida se purgó la columna con suero salino para evitar falsos resultados, basándonos en la forma correcta de tomar la presión venosa central de forma manual. En todas las medidas el paciente permaneció en decúbito supino, tomando como cero la línea medio axilar ${ }^{19}$. Se realizaron medidas en todas las ocasiones en las que el abdomen estuvo con volumen 0 , después de cada infusión y previo a todos los drenajes. En todas estas ocasiones la medición se hizo en reposo y en inspiración y espiración para calcular la PIP media (PIP inspiración + PIP espiración / 2). Utilizamos como valor referencia para el estudio la medida de PIP en reposo.

Se efectuaron 2 intercambios peritoneales con solución de glucosa al 2,3\% y 120 minutos de tiempo de permanecía.

En el primer intercambio se infundieron $2500 \mathrm{ml}$ de volumen de LD, durante la permanencia el paciente estuvo en bipedestación o sentado, posición en la que la PIP es máxima. El segundo intercambio se realizó con $1500 \mathrm{ml}$ de volumen de LD permaneciendo el paciente en reposo en cama durante los 120 minutos, para reproducir condiciones de PIP mínima.

El volumen de ultrafiltración se calculó por la diferencia de pesaje de las bolsas de infusión y de drenaje y la PIP en $\mathrm{cmH}_{2} \mathrm{O}$.

Finalizado el estudio en el hospital cada paciente continuó su tratamiento con la pauta de diálisis habitual.

Los valores recogidos se han expresado para la descripción estadística en porcentajes o medias, desviación estándar y rango, utilizando el paquete estadístico de Microsoft Office Excel. 


\section{Resultados}

Estudiamos a 42 pacientes, un $71,5 \%$ eran hombres, con una edad media de $59.31 \pm 1.23$ años, un IMC de $27.07 \pm 4.46$ y una SC de $1.819 \pm 0.19 \mathrm{~m}^{2}$. Todos nuestros pacientes toleraron el volumen máximo infundido y todos participaron en el estudio después de la oportuna información.

El volumen de infusión medio en el intercambio de $2500 \mathrm{ml}$ fue de $2554.76 \pm 94.37 \mathrm{ml}$ de LD, con un tiempo medio de permanencia de $120.4 \pm 2$ minutos y un drenaje de $2685 \pm 199.48 \mathrm{ml}$. En el intercambio de $1500 \mathrm{ml} \mathrm{la}$ infusión fue de $1504.76 \pm 30.49 \mathrm{ml}$ de LD con un tiempo medio de permanencia de $120.4 \pm$ 2.8 minutos y un drenaje de $1696.76 \pm 144.83 \mathrm{ml}$, los valores medios de la PIP con abdomen vacío tras 126 medidas fueron de $8.18 \pm 4.1 \mathrm{cmH}_{2} \mathrm{O}$ con un rango de $18.2 \mathrm{cmH}_{2} 0$; con el abdomen con un volumen de 1500 $\mathrm{ml}$ fueron de $11.2 \pm 4.2 \mathrm{cmH}_{2} 0$, rango $20 \mathrm{~cm} \mathrm{H}_{2} 0$; y con un volumen de $2500 \mathrm{ml}$ de $13.4 \pm 4.4 \mathrm{~cm} \mathrm{H}_{2} \mathrm{O}$, rango $19.5 \mathrm{cmH}_{2}$ 0. Tabla 1.

Tabla 1. Descripción de resultados de PIP y UF con diferentes volúmenes intraperitoneales.

\begin{tabular}{|c|c|c|c|c|c|c|c|}
\hline & PIP 0 & PIP 1,5 & PIP 2,5 & UF1,5 & UF\%1,5 & UF 2,5 & UF $\% 2,5$ \\
\hline MEDIA & 8,18 & 11,2 & 13,4 & 192 & $12,8 \%$ & 131 & $5,2 \%$ \\
\hline $\begin{array}{l}\text { DESVIACIÓN } \\
\text { ESTANDAR }\end{array}$ & 4,22 & 4,2 & 4,4 & 145 & $9,6 \%$ & 206 & $8,2 \%$ \\
\hline RANGO & $\begin{array}{l}18,2 \\
(18,-0,2)\end{array}$ & $\begin{array}{l}20 \\
(20,5,0,5)\end{array}$ & $\begin{array}{l}19,5 \\
(24,4,5)\end{array}$ & $\begin{array}{l}599 \\
(550,-49)\end{array}$ & $\begin{array}{l}39,9 \% \\
(36,7,3,3)\end{array}$ & $\begin{array}{l}950 \\
(500,-400)\end{array}$ & $\begin{array}{l}38 \% \\
(22,-16)\end{array}$ \\
\hline
\end{tabular}

PIP 0: Presión intraperitoneal con volumen intraperitoneal 0. PIP 1,5: Presión intraperitoneal con volumen intraperitoneal 1500 cc. PIP 2,5: Presión intraperitoneal con volumen intraperitoneal 2500 cc. UF 1,5: ultrafiltración conseguida con volumen intraperitoneal 1500 cc. UF 2,5: ultrafiltración conseguida con volumen intraperitoneal 2500 cc. UF\%1,5: porcentaje de ultrafiltración conseguida con volumen intraperitoneal 1500 cc. UF\%2,5: porcentaje de ultrafiltración conseguida con volumen intraperitoneal $2500 \mathrm{cc}$.

La UF media en el intercambio de $2500 \mathrm{ml}$ fue de $131 \pm 206 \mathrm{ml}$; en el intercambio de $1500 \mathrm{ml}$ fue $192 \pm 145 \mathrm{ml}$. Expresado en \% la UF media en el intercambio de mayor volumen sería de $5,2 \% \pm 8,2 \%$ y de $12,8 \% \pm 9,6 \%$ con volumen de $1500 \mathrm{ml}(p<0.001)$. (Figura 1).

No tuvimos ningún evento infeccioso, ni problemas con el catéter derivados de la manipulación del que fue objeto para el estudio.

\section{Discusión}

Medir la presión intraperitoneal en los pacientes en programa de diálisis peritoneal no es una práctica estandarizada, aunque diversos estudios confirman que conocer la PIP podría ayudar de forma importante a individualizar los tratamientos prescritos en nuestras unidades, y a detectar de forma precoz complicaciones derivadas de la terapia. Problemas de la pared abdominal como fugas o hernias, fallos en la UF o la no tolerancia a volúmenes altos en posición supina podrían relacionarse con aumentos de la PIP en muchos ca$\operatorname{sos}^{12,18,20}$.

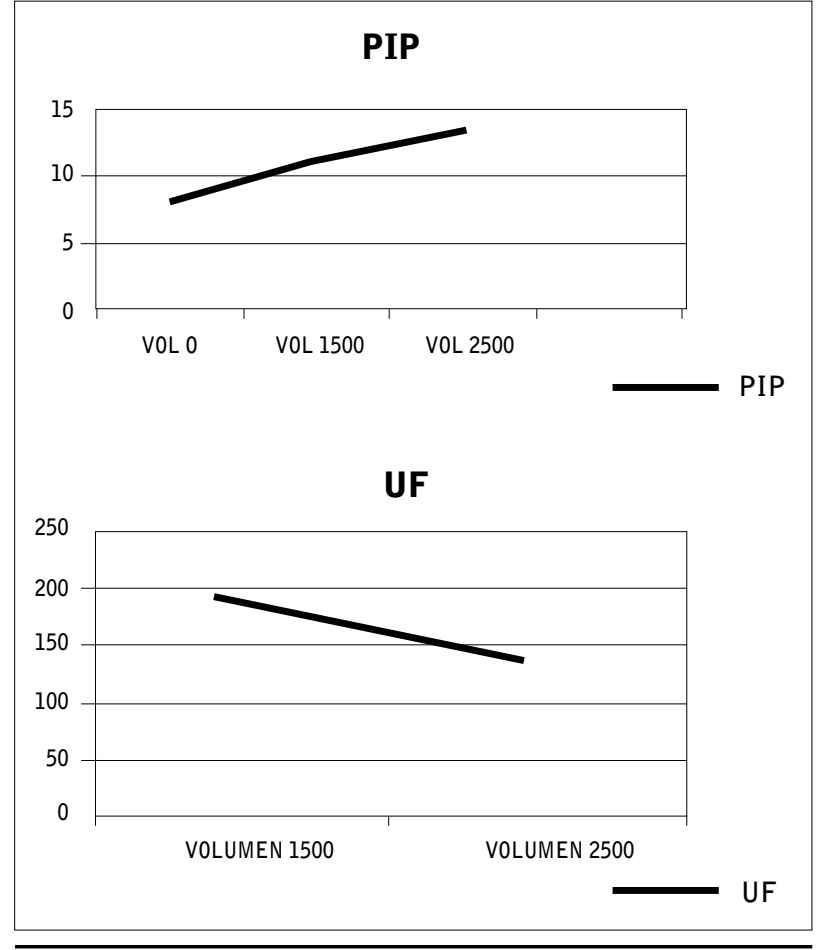

Figura 1. Gráficos de tendencia de PIP y UF con diferentes volúmenes.

PIP Presión intraperitoneal. UF Ultrafiltración. 
Nosotros, al igual que otros autores, hemos detectado un aumento de la PIP al aumentar el volumen de LD infundido. Con volúmenes de $2500 \mathrm{ml}$ de LD hemos obtenido PIP similares a las recogidas por otros autores como Durand en $1992^{7,13}$.

Se detectó una PIP superior a $20 \mathrm{~cm} \mathrm{H}_{2}$ O. en dos pacientes con un volumen de $1500 \mathrm{ml}$ y en cuatro pacientes con volúmenes de $2500 \mathrm{ml}$ pero en ningún caso tuvimos que acortar el tiempo de permanencia, ni abandonar el procedimiento, lo que coincide con otros autores con relación a la tolerancia de PIP elevadas ${ }^{20,21}$.

Los pacientes con IMC más alto tenían PIP más altas con volumen cero que pacientes con IMC más bajo $\mathrm{y}$ se repitió esta progresión en todos los volúmenes de llenado ${ }^{7,20}$.

Parece que un aumento de la PIP provocado por el aumento del volumen intraperitoneal influye de forma negativa en la UF, esto puede significar una influencia importante de la PIP en la UF y justifica que se piense en un aumento de la presión ante algunos fallos en la UF. Esta disminución de la UF podría deberse al aumento de la absorción linfática y de la filtración transtisular y a la disminución de la tasa de ultrafiltración transcapilar.

Tao Wang en contraposición a nuestros datos consigue mayor UF con volúmenes altos cuando los compara con volúmenes más pequeños, en su propio estudio justifica este cambio en la tendencia de otros estudios a la utilización de líquidos hipertónicos (glucosa de 3,86\%) que consiguen mantener el gradiente de concentración en niveles altos con permanencias de 120 minutos, esto nos hace pensar que también es importante analizar nuestros datos junto con datos del PET que nos indicarían el tiempo que cada paciente mantiene este gradiente de concentración ${ }^{22}$.

Después de la descripción de nuestros datos parece razonable pensar que es interesante contar con medidas de PIP unidas a datos facilitados por el PET, que nos informa de la pérdida de concentración de glucosa y del aclaramiento de urea y creatinina para individualizar la prescripción en DP.

\section{Conclusiones}

Podemos describir un aumento de la PIP al aumentar el volumen intraperitoneal, al mismo tiempo hemos observado una mayor UF con volúmenes más bajos que implicaban a su vez cifras menores de PIP.
Recibido: 2 septiembre 2016

Revisado: 18 septiembre 2016

Modificado: 10 octubre 2016

Aceptado: 21 octubre 2016

\section{Bibliografía}

1. Paniagua Sierra JR, Ventura García M de J, Vázquez Hernández R. Aumento de la presión intraabdominal y otras complicaciones. En Montenegro J, Correa Rotter R, Riella M. Tratado de Diálisis Peritoneal. Barcelona: Elsevier España SL; 2009. p. 349-360.

2. Al-Hwiesh A, Al-Mueilo S, Saeed I, Al-Muhanna FA. Intraperitoneal pressure and intra-abdominal pressure: are they the same? Perit Dial Int.2011 MayJun;31(3):315-9.

3. Tarwdowski ZJ, Khanna R, Nolph KD, et al. Intrabdominl pressures during natural activities in patines treated with continuos ambultory peritoneal diálisis. Nephron 1986; 44: 129-135.

4. Durand PY. Measurement of intraperitoneal pressure in PD patiens. Perit Dial Int 2005;25:333337.

5. Cheatham $M L$, Malbrain $M L$, Kirkpatrick $A$, Sugrue M, Parr M, De Waele J, Balogh Z, Leppäniemi A, Olvera C, Ivatury R, D'Amours S, Wendon J, HiIlman K,Wilmer A. Results from the International Conference of Experts on Intra-abdominal Hypertension and AbdominalCompartment Syndrome. Intensive Care Med. 2007 Jun;33(6):951-62.

6. Fischbach M. Hydrostatic intraperitoneal pressure: an objective tool for analyzing individual tolerance of intraperitoneal volume Perit Dial Int. Vol. 25, pp. 338-339.

7. Dejardin A, Robert A, Goffin E. Intraperitonel pressure in PD patients: relation-ship to intraperitoneal volume, body size and PD-related complications. Neprol Dial Transplant 2007; 22: 1437-1444. 
8. Durand PY, Chanliau J, Gambéroni J, Hestin D, Kessler M. Measurement of hydrostatic intraperitoneal pressure: a necessary routine test in peritoneal dialysis. Perit Dial Int. 1996;16 Suppl 1:S84-7.

9. Sánchez-Miralles A, Castellanos G, Badenes R, Conejero R. Síndrome compartimental abdominal y síndrome de distrés intestinal agudo. Med Intensiva 2013 - Vol. 37 Núm.2;37:99-109.

10. Blasco Cabañas C, Ponz Clemente $E$, Betancourt Castellanos L, Otero López S, Marquina Parra D, Gran Pueyo C, Moya Mejía C. Relación entre la presión intrabdominal en diálisis peritoneal con las hernias y fugas Enferm Nefrol 2012;15(2):94100.

11. Durand PY, Chanliau J, Gamberoni J, Hestin D, Kessler M. APD: Clinical Measurement of the Maximal Acceptable Intraperitoneal Volume. Avd Perit Dial, 1994;10: 63-7.

12. Gotloib L, Mines M, Garmizo L Varka I. Hemodynamic effects of increasing intraabdominal pressure in peritoneal dialysis. Perit Dial Bull, 1981 1: 41-43.

13. Durand PY, Chanliau I, Gamberoni I, Hestin D, Kessler $M$.Intraperitoneal Pressure, Peritoneal Permeability and Volume of Ultrafiltration In CAPD. Adv Perit Dial. 1992;8:22-5.

14. Flessner MF. Clinical importance of intraperitoneal pressure in peritoneal dialysis and measures to counteract its effect on net ultrafiltration. Adv Perit Dial. 1999;15:45-52.

15. Fesserner MF. Peritoneal ultrafiltration: physiology and failure. Contrib Nephrol 2009;163:7-14.
16. Imholz ALT, Koomen GCM, Voorn WJ, et al. Day to day variability of fluid and solute transport y upright and recumbent positions during CAPD. Nephrol Dial Transplant 1998; 13: 146-153.

17. Guías de Práctica Clínica en Diálisis Peritoneal. Sociedad española de Nefrología. Disponible en: http://www.senefro.org.

18. Fischbach M, Terzic J, Dangelser C, Schneider $P$, Roger ML, Geisert J. Improved Dialysis Dose By Optimizing Intraperitoneal Volume Prescription Thanks to Intraperitoneal Pressure Measurements in Children. Adv Perit Dial. 1997;13:271-3.

19. Durand PY, Chanliau J, Gamberoni J, Hestin $D$, Kessler M. Routine measurement of hydrostatic intraperitoneal pressure. Adv Perit Dial. 1992;8:108-12.

20. Di Lauro V, Luccio F, Colaluca M, De Francesco F, Pintauro A. Intraperitoneal hydrostatic pressure and volume in peritoneal dialysis patients. EDTNA ERCA J. 1999 Apr-Jun;25(2):16-7.

21. Harris KPG, Keogh AM, Alderson L. Peritoneal Dialysis fill volume: can the patient tell the difference? Perit Dial Int 2001; 21 (supl 3): 26-29.

22. Wang $T$, Heimburger 0 , Cheng $H$, Waniewski J, JonasBergstromJ, Lindholm B. Effect of increased dialysate fill volume on peritoneal fluid and solute transport. Kidney International, Vol. 1997; 52:1068-1076 